\title{
PREFACE
}

\section{In Memorial Issue of the late Professor Kenji Koga}

It is a great honor for me to contribute a preface for his memorial issue of Heterocycles. The impact of an organic chemist may be measured by his contributions to the discovery of the new materials and the pioneer of new chemistry field. Professor Koga has made this task the successful development in the fields of asymmetric synthesis and host-guest chemistry.

Professor Kenji Koga was born in Nagoya, February 11, 1938. Koga received his bachelor's degree in 1960 and his doctorate in 1967 both from the University of Tokyo under the guidance of Professor Shun-ichi Yamada in Pharmaceutical Sciences. He became Assistant Professor at the University of Tokyo in 1965 and then was promoted to Associate Professor in 1968. At his earlier research career he focused on the research of asymmetric synthesis utilizing amino acids as chiral sources such as stereoselective hydride reduction and stereocontrolled deamination of amino acid esters. Koga spent two years working with Professor D. J. Cram, the University of California at Los Angeles, where he contributed working host-guest recognition chemistry. In 1976, Koga promoted to full professor and developed extensively the amino acid chemistry which is the use of amino acids as chiral auxiliaries, particularly in asymmetric alkylation and conjugate addition as well as the structural elucidation of chiral lithium enolates. In addition, he also made an effort to clarify the direct evidence of the inclusion complexes utilizing cyclophanes for capture of organic guests.

Based on these contributions, Koga received numerous honors and awards as a pioneer in organic synthesis. In 1986, he received Inoue Prize for Science and the Pharmaceutical Society of Japan Award in 1994. In 1995, Koga received the Japan Academy Award in share with Professor S. Yamada on "New Synthetic Methodology for Optically Active Compounds Based on Asymmetric Transcription of Amino Acids."

To recognize his contributions to organic chemistry, the Japan Research Foundation for Optically Active Compounds has instituted an Award in his name, Yamada-Koga Prize in 2005.

With my best wishes to my best friend, the late Dr. Kenji Koga

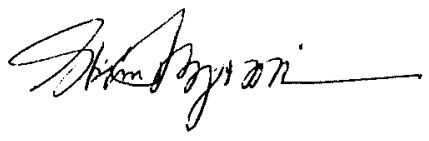

\author{
Shiro Ikegami \\ Professor, School of Pharmaceutical Sciences \\ Teikyo University
}

